\title{
Medicina Regenerativa para Úlceras Crónicas: Aplicación Cutánea de Células Troncales Mesenquimales de Gelatina de Wharton
}

\section{Regenerative Medicine for Chronic Ulcers: Cutaneous Application of Mesenchymal Stem Cells of Wharton's Jelly}

\author{
A. Parra-Barrera, C. M. Mejía-Barradas, C. C. Calzada-Mendoza, E. Mera-Jiménez, A. L. Sánchez-Corrales, \\ G. Gutiérrez-Iglesias \\ Escuela Superior de Medicina del Instituto Politécnico Nacional
}

\section{RESUMEN}

El empleo de células mesenquimales ha emergido como una parte importante en la medicina regenerativa con efectos positivos en la recuperación de tejidos dañados o perdidos. Las úlceras crónicas incluyen lesiones cutáneas que afectan a una gran parte de la población con diabetes mellitus, (por ejemplo 9.14\% desarrollan lesiones por pie diabético según ENSANUT 2016) y representan gastos económicos considerables, debido a la pobre calidad de vida que llevan los pacientes. El tratamiento convencional que se lleva a cabo en estos casos es a largo plazo, y los efectos benéficos generados que se presentan son limitados, ya que las lesiones reinciden por no contar con un tratamiento específico y regenerativo, solo paliativo. Es por ello que en este trabajo se obtuvieron y emplearon células troncales mesenquimales derivadas de gelatina de Wharton (CTM-GW) bajo los criterios propuestos por la Sociedad Internacional de la Terapia Celular. El tratamiento fue aplicado en dos pacientes con úlceras crónicas flebostáticas de diferentes condiciones médicas y se monitoreo a corto plazo, por lo que fue un estudio limitado. Los resultados mostraron que las CTM-GW indujeron la reconstrucción cutánea mediante formación de tejido de granulación, además de disminuir el proceso inflamatorio crónico en la zona de daño, lo que favoreció el índice de cierre de la herida. Por lo anterior, se propone la utilización de CTM-GW en úlceras flebostáticas crónicas como tratamiento en la regeneración de la estructura tisular con resultados a corto plazo.

PALABras Clave: Medicina Regenerativa; Células Troncales Mesenquimales de Gelatina de Wharton; Úlceras crónicas 


\begin{abstract}
Use of mesenchymal cells has emerged as an important part in regenerative medicine with positive effects in the recovery of damaged or lost tissues. Chronic ulcers include skin lesions that affect a large part of the population with diabetes mellitus, (for example, 9.14 develop diabetic foot according to ENSANUT 2016) and represent considerable economic expenses, due to poor quality of life in patients. The conventional treatment that is carried out in these cases is long term, and the generated beneficial effects that are presented are limited, since the injuries recur due to not having a specific and regenerative treatment, only palliative. That is why in this work they were obtained and used mesenchymal stem cells derived from Wharton's jelly (MSC-WJ) under the criteria proposed by the International Society for Cell Therapy. The treatment was applied in two patients with chronic ulcers phlebostatic of different medical conditions and short-term monitoring, so it was a limited study. The results showed that MSC-WJ induced skin reconstruction by tissue formation granulation, in addition to decreasing the chronic inflammatory process in the area of damage, which favored the index of wound healing. Therefore, the use of MSC-WJ in chronic phlebostatic ulcers is proposed as a treatment in the regeneration of the tissue structure with short-term results.
\end{abstract}

KEYwords: Regenerative Medicine; Mesenchymal Stem Cells; Chronic Ulcers

\section{Correspondencia}

DESTINATARIO: Gisela Gutiérrez Iglesias

INSTITUCIÓN: Escuela Superior de Medicina

del Instituto Politécnico Nacional

DIRECCIÓN: Salvador Díaz Mirón S/N, Col. Santo Tomás,

C. P. 11340, Ciudad de México, CDMX, México

CORREO ELECTRÓNICO: iglesiasgg@yahoo.com

\section{Fecha de recepción:}

30 de agosto de 2018

Fecha de aceptación:

16 de enero de 2019 


\section{INTRODUCCIÓN}

Las úlceras crónicas (UC), son alteraciones en la piel que toman más de seis semanas en cicatrizar ${ }^{[1]}$. El envejecimiento, la diabetes y la obesidad incrementan la prevalencia de las UC, por lo que son una de las principales causas de morbilidad y mortalidad ${ }^{[2]}$. Son más frecuentes en personas de la tercera edad con enfermedad vascular periférica ${ }^{[3]} \mathrm{y}$ se caracterizan por la presencia de dolor ${ }^{[4]}$ e incapacidad para cicatrizar ${ }^{[5]}$.

Aunque se han desarrollado tratamientos para este tipo de problemas, la mayoría de ellos no tiene efectos benéficos a corto plazo, lo que repercute sobre el gasto público de las instituciones de salud pública y sobre la calidad de vida de los pacientes ${ }^{[6]}$. Por otro lado, la medicina regenerativa y la ingeniería de tejidos ofrecen una alternativa en terapias celulares para el tratamiento de este tipo de lesiones ${ }^{[7]}$ debido a que pueden reparar/reemplazar el tejido dañado ${ }^{[8]}$. Las células troncales mesenquimales (CTM) son las células más empleadas en medicina regenerativa debido a que son células progenitoras con capacidad de autoregenerarse y multipotentes, pueden aislarse de varios tejidos como: médula ósea ${ }^{[9]}$, tejido adiposo ${ }^{[10]}$, sangre de cordón umbilical ${ }^{[11]}$, sangre periférica ${ }^{[12]}$, endometrio ${ }^{[13]}$, pulpa dental ${ }^{[14]}$, líquido ammiotico ${ }^{[15]} \mathrm{y}$ tejido tumoral ${ }^{[16]}$. Estas células pueden caracterizarse con base en los criterios propuestos por la International Society for Cell Therapy (ISCT) e incluyen: adherencia al sustrato de cultivo, morfología fibroblastoide, con la capacidad para diferenciarse en células especializadas de la capa mesodérmica: adipocitos, condrocitos, osteoblastos [15], expresión de antígenos membranales: CD73, CD9o y CD105, así como ausencia de los antígenos como: CD34, CD45 y HLA-clase II [9, 10, 17].

Actualmente, se han aplicado en terapias celulares para disminuir la enfermedad injerto contra huésped o GvDH ${ }^{[18]}$, regeneración epitelial en colon ${ }^{[19]}$, ulceras crónicas ${ }^{[20,21]}$, ulceras asociadas a esclerosis sistémica ${ }^{[8]}$ colitis ulcerativa ${ }^{[2]}$ y pie diabético ${ }^{[23]}$. Sin embargo, pocos estudios han realizado la aplicación de Células Troncales Mesenquimales derivadas de Gelatina de Wharton para el tratamiento de ulceras crónicas [24]. Por ello, en este trabajo se realizó la aplicación de CTM derivadas de gelatina de Wharton en dos pacientes con diagnóstico de úlcera crónica flebostática, donde se analizaron las modificaciones estructurales del tejido mediante técnicas histológicas, área de la herida, índice de cierre antes y después del tratamiento.

\section{METODOLOGÍA}

\section{Presentación de los casos}

Caso 1: Paciente femenina, 55 años de edad con diabetes mellitus tipo 2, insuficiencia venosa periférica crónica y úlcera flebostática en región maleolar del miembro pélvico derecho, más de 3 años de evolución. Caso 2: Paciente femenina, 56 años de edad con insuficiencia venosa periférica crónica, úlcera flebostática en región maleolar del miembro pélvico derecho, más de 5 años de evolución con falla de injerto autólogo.

\section{Recolección cordones umbilicales}

La selección de cordones umbilicales se realizó en base a los criterios establecidos por la Ley General de Salud Mexicana, la Norma Oficial Mexicana NOM-253SSA1-2012 y por el Banco de Sangre de Cordón Umbilical del Instituto Mexicano del Seguro Social Centro Médico Nacional "La Raza". Una vez seleccionadas las posibles donadoras y previa firma del consentimiento informado, se realizó la recolección de los cordones en condiciones de esterilidad, en una solución amortiguada de fosfatos o PBS ( $\mathrm{NaCl} 8.06 \mathrm{~g}, \mathrm{KCl} 0.22 \mathrm{~g}, \mathrm{Na}_{2} \mathrm{HPO}_{4} 1.15$ g, $\mathrm{KH}_{2} \mathrm{PO}_{4} 0.20 \mathrm{~g} \mathrm{H}_{2} \mathrm{O} 1000 \mathrm{~mL}^{-1}$ ) y se mantuvieron a 4 ${ }^{\circ} \mathrm{C}$ hasta la extracción de las CTM-GW.

\section{Aislamiento de las CTM-GW}

El cordón se cortó en secciones de aprox. 3mm, fueron eliminadas las arterias y vena umbilicales, se disgregó enzimáticamente primero con colagenasa tipo I al $1 \%$ en PBS (Sigma-Aldrich, USA) y posteriormente 
con tripsina al $0.1 \%$, en agitación constante a $37.5{ }^{\circ} \mathrm{C}$ por 60 y 30 minutos respectivamente. El sobrenadante, fue centrifugado a $180 \mathrm{~g} / 10 \mathrm{~min}$, el botón celular se resuspendió en medio DMEM-F12 complementado con $10 \%$ de suero fetal bovino y 1\% de antibiótico-antimicótico (Gibco ${ }^{\circledR}$, USA). Las células fueron cultivadas en cajas Petri de $100 \mathrm{~mm}$ x $20 \mathrm{~mm}$ (Corning Centri Star ${ }^{\mathrm{TM}}$, USA), se mantuvieron a $37^{\circ} \mathrm{C}$ en una atmósfera con humedad saturada con $5 \%$ de $\mathrm{CO}_{2}$ en incubadora modelo Galaxy 170R (R.T.L., Colombia). Cuando los cultivos tuvieron una confluencia del 80 - 90\%, se realizó la resiembra de las mismas (pases), este procedimiento se repitió hasta obtener las células en un pase no mayor a 6 para poder caracterizar las células y aplicarlas en los pacientes.

\section{Caracterización de las CTM-GW}

Se realizó tomando en cuenta los criterios propuestos por la ISCT como: 1) Morfología fibroblastoide y adherencia; las células de Gelatina de Wharton humana se mantuvieron en observación mediante el uso de un microscopio Oxion Inverso (PCE Instruments, Chile), se realizó la documentación fotográfica con una cámara digital Samsung-ST150F 16.2-Megapixel (modelo: EC-ST150FBPBUS). La adherencia se determinó durante los cambios de medio, las células adheridas permanecieron en las cajas Petri en tanto, las células en suspensión, fueron desechadas durante este procedimiento; 2) Pruebas de plasticidad celular. Para inducir la diferenciación hacia osteoblastos y adipocitos, las CTM-GW se cultivaron con Stempro ${ }^{\circledR}$ Osteogenesis Differentiation y Stempro ${ }^{\circledR}$ Adipogenesis Differentiation (USA) durante 21 días. Para comprobar la diferenciación se realizó: a) Tinción con rojo de alizarina S (1,2-dihidroxiantraquinona) (Merck Mlipore, USA): las células se lavaron con PBS y fijaron con formol al 10\% durante 10 minutos, en seguida se agregó rojo de alizarina $S$ (1,2-dihidroxiantraquinona) durante 10 minutos, se realizaron dos lavados con PBS. b) Tinción con rojo oleoso: las células se fijaron con formol al 10\%, tiñeron con rojo Oleoso (Sigma-Aldrich,
USA) en isopropanol al $60 \%$ (Sigma-Aldrich, USA) por $10 \mathrm{~min}$, y se lavaron en dos ocasiones con isopropanol al 60\%. 3) Expresión de antígenos de superficie; las células se lavaron con PBS y se les agregó EDTA (ácido etildiaminotetraacético) 2mM (Research Organics, USA) para desprenderlas mediante raspado. Una vez en suspensión con $\mathrm{NaCl} 0.9$ \% se incubaron con los siguientes anticuerpos anti-humano; anti-HLA-DR (cat. sc-3939); anti-CD34 (cat. Sc-343503); anti-CD45 (cat. MA1-12219, Thermo Scientific); anti-CD105 (cat. 323204); anti-CD73 (cat. 344004) y anti-CD9o (cat. 328108, Biolegend), lavaron con PBS y centrifugaron a $3000 \mathrm{rpm}$ por $1 \mathrm{~min}$. El botón celular se resuspendió en paraformaldehído (Sigma-Aldrich, USA) al $2 \%$, la lectura se realizó en un citómetro de flujo FACScalibur (Becton Dickinson, USA). Los resultados obtenidos se reportaron como porcentaje de células positivas para cada marcador tomando como 100 \% el número total de eventos analizados.

\section{Separación en etapas de estudio}

El área de las heridas y el consumo de antiinflamatorios no esteroideos fueron estudiados en tres etapas diferentes: terapia convencional (TC); desde el momento de la valoración inicial (semana -6) al inicio del tratamiento (semana 0), 1er Dosis (1er-D); desde el inicio del tratamiento (semana 0) hasta la segunda aplicación (semana 4), y finalmente, 2da Dosis (2da-D); que abarcó desde el momento de la segunda aplicación (semana 4) hasta que las pacientes abandonaron el tratamiento (semana 9 aproximadamente).

\section{Aplicación de CTM-GW en pacientes}

Para realizar la aplicación de las CTM-GW en los pacientes se realizaron los siguientes pasos; 1) Cuantificación del área de la herida. Para calcular el área de daño se obtuvieron imágenes con una cámara digital Samsung-ST150F 16.2-Megapixel (modelo: EC-ST150FBPBUS) y el área se determinó con el programa Image $1.46 \mathrm{r}$, los valores obtenidos fueron expresados en centímetros cuadrados $\left.\left(\mathrm{cm}^{2}\right), 2\right)$ 
Preparación de la herida. Se realizó la curación con jabón quirúrgico neutro Antibenzil (1 g de cloruro de benzalconio $\cdot 100 \mathrm{~mL}^{-1}$ de vehículo) (Altamirano farmacéuticos), solución salina al 0.9\% (PISA farmacéutica), 3) Preparación de las células para su aplicación: Las CTM-GW fueron despegadas con tripsina/EDTA (Sigma-Aldrich, USA), y se aplicaron 100,000 $\pm 10,000$ células $\cdot \mathrm{cm}^{-2}$ de herida, 4) Colocación del Surgicel ${ }^{\circledR}$ (Ethicon, USA): en el caso 2 se colocó una malla de celulosa regenerada oxidada absorbible $35 \times 5 \mathrm{~cm}^{2}$ a la herida mediante puntos quirúrgicos con sutura Catgut crómico 3/0 (Ethicon, USA), 5) Aplicación de las $C T M-G W$. Se realizó directo en la herida mediante infiltración intradérmica e irrigación en el lecho de la herida en dos ocasiones, el día de la primera aplicación se consideró como día o y la segunda dosis se realizó a la cuarta semana, por último las lesiones se protegieron con un apósito hidrocoloide DuoDERM ${ }^{\circledR}$ (ConvaTec, Nordic Capital y Avista Capital Partners), gasas y apósitos estériles hasta la valoración de las mismas.

\section{Histología}

Los cortes histológicos fueron teñidos con Hematoxilina y Eosina (H-E), las biopsias se tomaron antes de iniciar el tratamiento y a los 30 días de la primera aplicación, fijaron con formol al $10 \%$ amortiguado (formaldehido 4\%, NaH2PO4.H2O $4 \mathrm{~g}$, $\mathrm{Na}_{2} \mathrm{HPO}_{4} \cdot 7 \mathrm{H}_{2} \mathrm{O} 6.5 \mathrm{~g} \cdot \mathrm{H} 2 \mathrm{O} 1000 \mathrm{~mL}^{-1}$ ), fueron incluidos en parafina, se realizaron cortes semi-finos de 4-7 $\mu \mathrm{m}$, se montaron en portaobjetos, Tinción por Hematoxilina y Eosina: las laminillas con los tejidos se desparafinaron, hidrataron y tiñeron con Hematoxilina de Harris. Posteriormente, las laminillas se lavaron con agua corriente, contrastaron con eosina, deshidrataron, aclararon y montaron con resina de metacrilato/ Acrilato (Entellan ${ }^{\circledR}$ Merck, Alemania).

\section{Respuesta de la lesión}

La recuperación de la lesión fue determinada al plantear el índice úlcera, que se interpretó por el porcentaje del área de la herida abierta remanente con respecto al área de la herida al inicio del tratamiento. También se expresó la tasa de recuperación ( $\mathrm{T}_{\mathrm{r}}$ ) de la herida como el cociente $\mathbf{T}_{\mathbf{r}}=\mathbf{A}_{\mathbf{r}} / \mathbf{t}_{\mathrm{r}}$, donde $\mathbf{A}_{\mathrm{r}}$ es el valor del área recuperada $\left(\mathrm{cm}^{2}\right)$ durante el tratamiento, $\mathrm{y} \mathrm{t}_{\mathrm{r}}$ son los días que duró la recuperación del área. Las extensiones de las áreas ulceradas y recuperadas fueron evaluadas a través del análisis de imágenes con el programa ImageJ.

\section{Aspectos bioéticos}

El comité de bioética local autorizó este trabajo así como el método de selección de los sujetos de estudio, cumplió con los principios y valores bioéticos de beneficencia, respeto a las personas y equidad.

\section{Análisis estadístico}

El análisis de las áreas se realizó mediante la prueba estadística ANOVA de una vía con el programa Microsoft Excel.

\section{RESULTADOS}

\section{Caracterización celular}

Morfología fibroblastoide y adherencia a las placas de cultivo: las células aisladas mostraron una morfología alargada que asemejaba a fibroblastos, característica relacionada con la adherencia de las CTM a la placa de cultivo (figura 1A).

Diferenciación osteogénica: los cultivos mostraron positividad para la tinción de rojo de Alizarina, lo que indica la presencia de $\mathrm{Ca}++\mathrm{y}$ fosfatasa alcalina como distintivo de las células mesenquimales diferenciadas a osteoblastos (figura1B).

Diferenciación adipogénica: en los cultivos mantenidos con medio adipogénico se pudieron apreciar pequeñas vesículas lipídicas en el citoplasma, positivas para la tinción de rojo Oleoso, específico para los adipocitos (figura 1C). Expresión de antígenos de superficie: los marcadores de superficie que se analizaron para las células aisladas fueron CD90/CD73 
(93.38\% de positividad) y CD105 (35.98\% de células positivas), mientras que la expresión de antígenos hematopoyéticos HLA-DR, CD34 y CD45 estuvo prácticamente ausente (0.04 y $0.16 \%$, respectivamente) en los cultivos (figura 1D). Con ello se cumplieron los criterios propuestos por la ISCT.

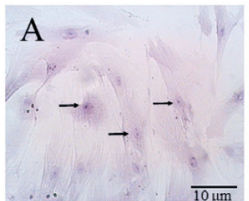

D $\quad \mathrm{CAF}$

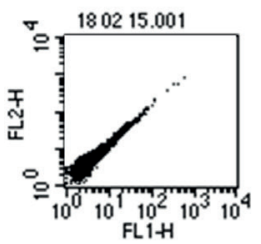

HLA-DR

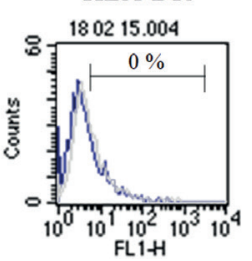

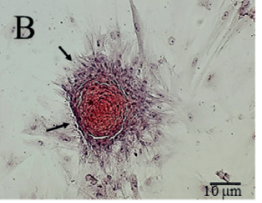

CD90/CD73

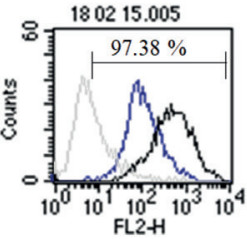

CD34
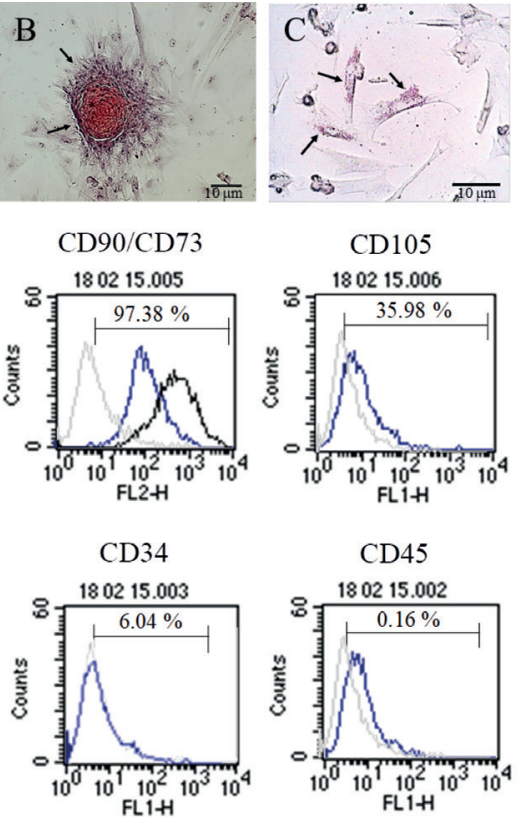

CD105

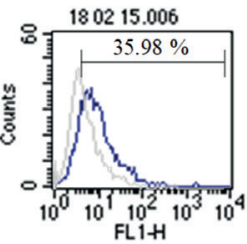

CD45
FIGURA 1. Obtención y caracterización de las CMM-GW.

(A) Cultivos celulares en $6^{\circ}$ pase, $(\uparrow)$ células de forma fibroblastoide. (B) Células en medio de diferenciación osteogénica, $(\uparrow)$ acúmulos de $\mathrm{Ca}++$ y fosfato extracelular teñidas con rojo de alizarina. (C) Células en medio de diferenciación adipogénico, $(\uparrow)$ con vesículas lipídicas intracelulares teñidas con rojo oleoso. (D) Análisis de la expresión de proteínas hematopoyéticas de superficie por citometría de flujo de 10 mil eventos celulares.

\section{Respuesta de la lesión hacia el tratamiento}

Caso 1; la figura 2A muestra la herida al día o del tratamiento, donde se puede observar un estado eritematoso y exudativo, sin evidente proceso de infección en la zona, por otro lado, la figura 2B obtenida al séptimo día de la primera aplicación de las mesenquimales donde es posible apreciar la formación de tejido en el lecho de la misma. Caso 2; la figura 2C muestra el área de lesión recubierta por la malla de Surgicel ${ }^{\circledR}$ al inicio de la aplicación celular. En la figura 2D se aprecia la formación de tejido sobre la malla colocada al inicio del tratamiento, con lo que se disminuyó la profundidad de la lesión. Para representar el beneficio del tratamiento con células solamente y células en Surgicel ${ }^{\circledR}$, se llevó a cabo un análisis de índice de úlcera y tasa de recuperación, las cuales se describen a continuación.
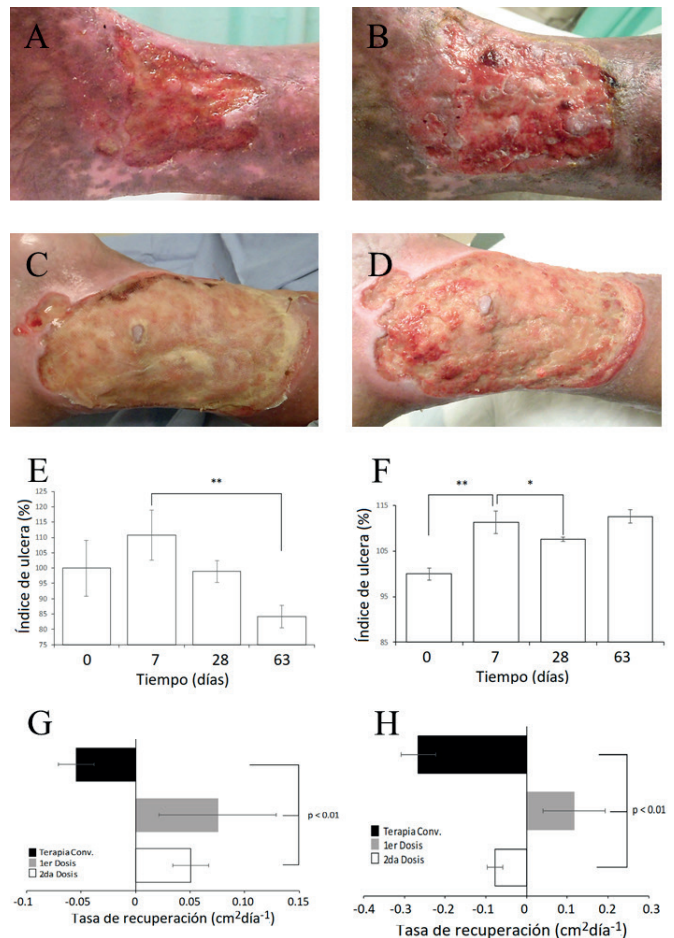

FIGURA 2. Respuesta de la lesión para Caso 1 (A, B, E y 299 G) y Caso 2 (C, D, F y H). Aspecto de la herida al inicio 300 del tratamiento (A y C), aspecto de la herida a los 7 días 301 de la primera aplicación del tratamiento (B y D), índice 302 de úlcera (E y F), tasa recuperación (G y H).

\section{Índice de úlcera y tasa de recuperación}

En el caso 1; durante el periodo de observación se pudo apreciar un incremento en el porcentaje del área al día 7 (figura 2E), sin embargo, al término del tratamiento (día 63), la herida mostro una disminución del área de lesión. Por otro lado, con el análisis de las áreas de herida se calculó la tasa de recuperación de herida, en el cual se demostró que con el tratamiento convencional este valor era negativo (-0.05), lo que señala un 
aumento en el área afectada. Posteriormente, con el tratamiento de células mesenquimales, la tasa de recuperación mostró valores significativamente positivos (0.05 y 0.10), lo que indica un índice de recuperación de herida favorable.

En el caso 2 esta paciente mostró un área de herida incrementada con el tratamiento convencional (figura $2 \mathrm{~F}$, día 0); e igual que pasó en el caso 1 , el índice de úlcera aumentó al día 7; no obstante, en el día 28 posterior a la primera aplicación el índice de úlcera alcanzó a disminuir y se mantuvo en el tiempo hasta el día 63 (figura 2F). Por otro lado, el análisis de la tasa de la recuperación de la herida mostró valores negativos con el tratamiento convencional (0.3, figura $2 \mathrm{H}$ ) y aunque alcanzó valores positivos (0.15 en la primer dosis), subsecuentemente se reflejó en un valor negativo en la tasa de cierre; sin embargo, éste número negativo se mantuvo menor al 0.1, que fue menor al que se presentó al inicio del tratamiento (figura $2 \mathrm{H}$ ), lo que refleja que con el tratamiento número 2 las condiciones de úlcera mejoraron con el material Surgicel ${ }^{\circledR}$.

\section{Análisis histológico de las úlceras antes y después del tratamiento}

En los resultados de las tinciones por H\&E para el caso 1; fue posible apreciar previo al tratamiento, un infiltrado inflamatorio perivascular así como microangiopatía (3A, flechas) y las fibras depositadas en el área de lesión (3A, asteriscos). Después de administrarse la aplicación de células mesenquimales (figura 3B), el infiltrado inflamatorio disminuyó (3B, flechas) y las fibras de colágeno fueron más abundantes (3B, asteriscos), delimitando los nuevos vasos sanguíneos que son perceptibles en la tinción (3B, cabezas de flecha obscuras). En el caso 2, previo a la aplicación de las células mesenquimales (figura $3 \mathrm{C}$ ), se aprecian extensas zonas de necrosis (3C, estrellas), edema (3C, cabezas de flecha claras), infiltrado inflamatorio perivascular, vasculitis, microangiopatía (3C, asteriscos) y microcoágulos de fibrina en vasos sanguíneos. En esta paciente se presentaron mayores beneficios por el tratamiento de la ulcera con las células mesenquimales y Surgicel ${ }^{\circledR}$, que fueron observados en los cortes histológicos (figura 3D); donde la zona de necrosis fue desplazada (3D estrellas) por un aumento de células que conformaron una zona de proliferación vascular. Debido a lo anterior, la conformación del tejido puede verse más sólido, como se mostró en las fotos de la lesión de la figura 2.
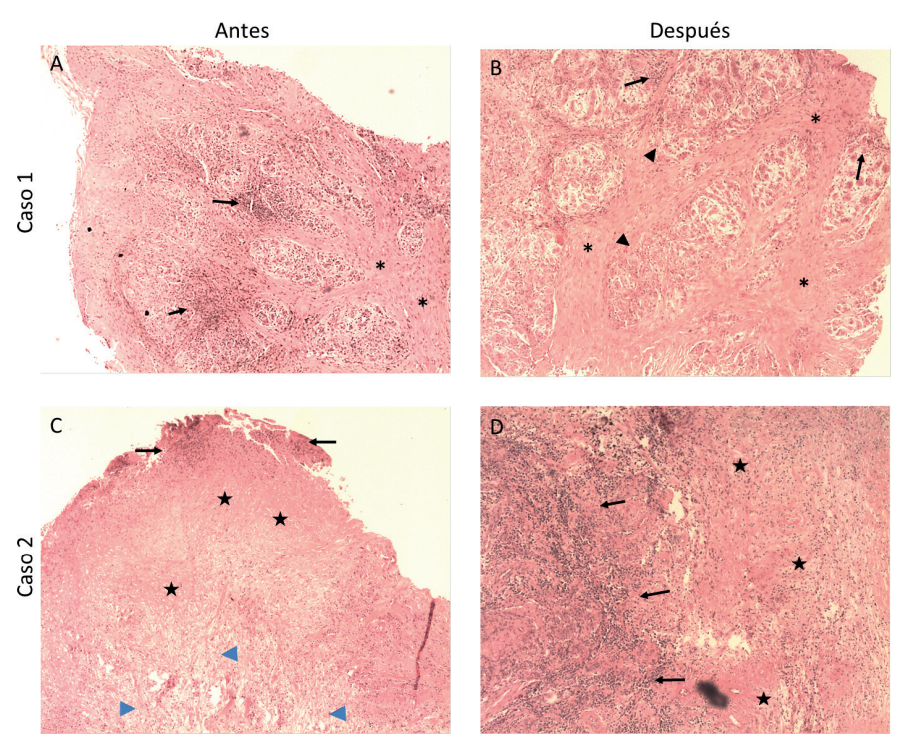

FIGURA 3. Cortes histológicos de las lesiones antes (A, C) 319 y después $(B, D)$ del tratamiento para Caso 1 (panel 320 superior) y Caso 2 (panel inferior). ( $\uparrow$ ) infiltrado 321 linfocitario perivascular, (*) fibras de colagena, $(\boldsymbol{\Delta})$ vaso 322 sanguíneo, $(\Delta)$ edema, $(\star)$ necrosis.

\section{DISCUSIÓN}

El desarrollo de estrategias de medicina regenerativa o terapia celular con células troncales mesenquimales ha demostrado tener resultados a corto plazo, además de aminorar los costos en el sector salud ${ }^{[25,26]}$. Para ello es importante que la obtención no sea invasiva, que sea posible su trasplante autólogo o alogénico, que el aislamiento y cultivo se basen en las buenas prácticas de manufactura y por último, cumplir con los postulados propuestos por la sociedad internacional para la terapia celular (ISCT) ${ }^{[27]}$. 
Las úlceras son lesiones cutáneas de tamaño y forma variable que persisten por más de 6 semanas que no responden a tratamiento convencional, por lo que se transforman en crónicas ${ }^{[28]}$. Estas heridas se caracterizan por presentar alteraciones en la estructura microvascular que se favorece por diferentes comorbilidades metabólicas preexistentes, y por lo tanto, su tratamiento resulta difícil, largo y costoso, afectando de forma importante la calidad de vida del paciente ${ }^{[28-30]}$.

En este trabajo se aplicó terapia regenerativa a partir de CTM derivadas de gelatina de Wharton (tejido de cordón umbilical de obtención no invasiva) en úlceras crónicas. Las CTM fueron aisladas y caracterizadas en base a los criterios propuestos por la ISCT [27]. Primeramente, la naturaleza troncal de las células fue evidenciada por presentar morfología de tipo fibroblastoide, adherencia a los recipientes de cultivo, capacidad de diferenciación tanto osteogénica como adipogénica, y se identificaron los antígenos de superficie CD90, CD73 y CD105, por lo que los cultivos utilizados en este estudio cumplieron los criterios para ser utilizados en terapia ${ }^{[27]}$.

Las ulceras crónicas de pacientes con diabetes mellitus recientemente han sido tratadas con CTM de diferentes fuentes como M.O. ${ }^{[31,32]}$, tejido adiposo ${ }^{[33,34,35]} \mathrm{y}$ piel ${ }^{[36]}$. En este trabajo, se confirmó lo reportado previamente por muchos investigadores, donde las CTM suprimen la inflamación excesiva, estimulan la angiogénesis de novo, facilitan la cicatrización y reducen el tamaño de la herida, por lo que brinda enormes posibilidades para la sanación de dichas úlceras [37]. Las características histológicas macroscópicas y físicas de la herida (inflamación evidente, apertura de herida) después de la primera aplicación, mostraron la formación de tejido de granulación y fibrótico al séptimo día del tratamiento con las CTMs. La cicatrización fue más evidentes en el caso 2 (figura 2c y d), donde se utilizó la malla de Surgicel ${ }^{\circledR}{ }^{[38]}$. Se pudo observar un efecto aditivo de las CTM con el Surgicel. Para éste último, se han descrito los beneficios en casos de úlceras pero sin aplicación de CTM ${ }^{\text {[39] }}$, por lo que al utilizarlo en conjunto con las CTM tuvo mayores beneficios. Chen y cols., (2015) ${ }^{[40]}$ en un modelo de ratón, demostraron que las CTM-MO en combinación con un hidrogel n-isopropilamida (NIPAM) a los 35 días post-implante una disminución de la inflamación, formación de tejido de granulación, aumento de macrófagos M2, angiogénesis y regeneración tisular.

En nuestro trabajo, a los 65 días post-tratamiento encontramos que disminuye el tamaño de la lesión y presencia de formación de vasos sanguíneos. Cabe señalar que el tamaño de las lesiones en el modelo murino son muy pequeñas (aproximadamente $0.5 \mathrm{~cm}^{2}$ ) a comparación de las lesiones de nuestros pacientes (aproximadamente $10 \times 7 \mathrm{~cm}^{2}$ ) por lo que la diferencia es muy marcada entre ambos grupos de trabajo ${ }^{[40]}$.

Las CTM estimulan diferentes mecanismos moleculares y celulares ${ }^{[41-44]}$, por ejemplo: a) Se promovió la diferenciación de las CTM a diferentes tipos celulares ${ }^{[41]}$ como células endoteliales ${ }^{[29]}$, pericitos ${ }^{[45]}$, musculo liso ${ }^{[46]}$, fibroblastos ${ }^{[47-49]}$, miofibroblastos ${ }^{[50,51]}$ o queratinocitos ${ }^{[52]}$, todas ellas indispensables en los procesos

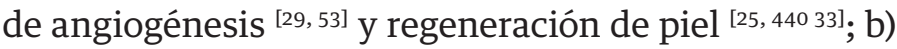
probable liberación de diferentes factores de crecimiento y citocinas que promueven la proliferación de las células residentes ${ }^{[41]}$ y c) las CTM reestructuraron el microambiente, mejorando las condiciones para la regeneración tisular ${ }^{[41]}$.

Ya que las úlceras crónicas presentan sucesiones continuas de inflamación (afectando la formación de tejido y remodelación), no se concluye su cicatrización o regeneración, ocasionando que se retrase el cierre de la herida ${ }^{[54]}$. En este estudio, los análisis histopatológicos iniciales para ambos casos, mostraron procesos inflamatorios crónicos como microangiopatía y zonas de fibrosis y necrosis (típico de dichas lesiones) ${ }^{[54-56]}$; sin embargo, a partir del tratamiento con las CTM fue 
posible apreciar en los dos casos una disminución de la cantidad de linfocitos infiltrantes, que se relaciona a la capacidad inmunomoduladora de las células mesenquimales en presencia de ambientes inflamatorios crónicos, permitiendo así la regeneración de piel ${ }^{[41,52 \text {, }}$ 54]. Lo anterior conlleva a un incremento en la cantidad y calidad de vasos sanguíneos formados ${ }^{[57,58]}$, los cuales también fueron observados en los cortes histológicos de las pacientes con tratamiento. El efecto que se mostró a corto plazo al aplicar las CTM-GW sobre el cierre de las heridas crónicas, concuerda con los resultados de Navone (2014) ${ }^{[59]}$ quien utilizó un andamio de fibroína y CTM, donde la herida se reduce en un $40 \%$. Kazohide M., y colaboradores (2015) ${ }^{[60]}$ utilizaron células sobre un hidrogel de ácido hialurónico al 4\% y se redujo la ulcera a los 28 días post-tratamiento (similar a nuestros resultados). Por otro lado, el grupo de Wu y cols (2017) ${ }^{[61]}$ demostró que el uso de CTM derivadas de placenta disminuyeron el tamaño de las lesiones en $51 \%$ de los pacientes en un plazo mayor que este estudio ( 3 meses pos-trasplante). Ribeiro y cols., (2014) ${ }^{[62]}$ observaron que las CTM-GW $\left(1 \times 105 / \mathrm{cm}^{2}\right)$ incluidas en un hidrogel de poli-vinil alcohol, a los 60 días post-tratamiento la herida sana al $100 \%$, así los cortes histológicos mostraron reepitelización, tejido de granulación y formación de vasos sanguíneos en la herida. En este trabajo solo muestra reducción de la herida y la formación de vasos sanguíneos. Sin embargo hay que destacar que las ulceras crónicas son de pacientes diabéticos e insuficiencia venosa periférica crónica con más de 3 y 5 años de evolución ${ }^{[62]}$.

Al realizar el análisis del área de la herida, se pudo observar una apertura considerable en la lesión que llevaba largo tiempo con terapia convencional, lo que indica que ésta no fue suficiente para favorecer el cie- rre; sin embargo, después de la primera dosis se pudo apreciar una disminución del área que se mantuvo en el tiempo. Esta mejoría pudiera deberse al efecto de las citocinas secretadas por las células mesenquimales sobre los fibroblastos o miofibroblastos ${ }^{[50]}$, además de los otros efectos de las CTM ya mencionados. Aunque el análisis de la herida mostró mejoría debido al tratamiento, la lesión no se recuperó en su totalidad; nosotros proponemos ajustar a una mayor dosis celular y al menos otras dos aplicaciones, sobre todo por la extensión de la lesión. Por otro lado, en el caso 2 se demostró que la aplicación de células en conjunto con materiales que promueven el soporte celular (en este caso utilizamos un producto derivado de la oxidación de la celulosa) ${ }^{[38]}$, es una mejor alternativa para recuperar el tejido en menor tiempo, por lo que la medicina regenerativa en este tipo de lesiones, pueda dar paso al siguiente nivel, que es la "ingeniería de tejidos", donde se puede utilizar la piel autóloga, alogénica o sintética [25, 52], así como biomateriales, con el fin de potencializar la resolución de las heridas flebostáticas crónicas.

\section{CONCLUSIONES}

La terapia celular con aplicación de CTM provenientes de gelatina de Wharton sobre úlceras flebostáticas de difícil manejo, es adecuada y representa una interesante alternativa médica por sus resultados a corto plazo, y podría aplicarse en otros tipos de lesiones. Además de lo anterior, la utilización de materiales como el producto de la oxidación de la celulosa, permite una mejor regulación de los procesos inmunológicos y modeladores del tejido; esto abre las puertas para que en este tipo de lesiones se aplique la ingeniería de tejidos en conjunto con células troncales. Lo anterior, también representaría una reducción del tiempo de convalecencia. 


\section{REFERENCIAS}

[1] Nelson EA, Adderley U. (2016). Venous leg ulcers. BMJ Clin Evid. Jan 15; 2016. DOI: 1902 [pii]

[2] Kelly T., et al., (2008). Global burden of obesity in 2005 and projections to 2030. Int J Obes (Lond). 32(9):1431-7. DOI: 10.1038/ijo.2008.102, ijo2008102 [pii]

[3] Aberdam D., (2004). Derivation of keratinocyte progenitor cells and skin formation from embryonic stem cells. Int J Dev Biol. 48(23):203-6. DOI: 10.1387/ijdb.031760da, 031760da [pii]

[4] Agale SV. (2013). Chronic Leg Ulcers: Epidemiology, Aetiopathogenesis, and Management. Ulcers. 2013:9. DOI: $10.1155 / 2013 / 413604$

[5] Alzahrani H., et al., (2013). Healing of chronic diabetic foot ulcers with a skin substitute: Patient selection is the key to success. Open Journal of Regenerative Medicine. 2:15-9.

DOI: $10.4236 /$ ojrm.2013.22003

[6] Antunez G.F.T., et al., (2008). Tratamiento combinado para úlcera venosa crónica: compresión, escleroterapia de perforantes guiada por ultrasonido e injerto de piel. Revista Mexicana de Angiología [serial on the Internet]. 36(2): Available from: https://goo.gl/ordNPF

[7] Atala A, et al., (2012). Engineering complex tissues. Sci Transl Med. 14;4(160):160rv12. DOI: 10.1126/scitranslmed.3004890

[8] Marfia G, et al., (2015). Mesenchymal stem cells: potential for therapy and treatment of chronic non-healing skin wounds. Organogenesis. 2015;11(4):183-206. DOI: 10.1080/15476278.2015.1126018

[9] Acevedo-Olvera L.F., (2016). Effect of dehydroepiandrosterone on expression of BMP2, SPARC and RUNX2 in human bone marrow mesenchymal stem cells. Revista Mexicana de Ingeniería Química 15(1): http://www.redalyc.org/pdf/620/62045307005.pdf

[10] Parra-Barrera A., et al., (2017). Comparación entre Células Troncales Mesenquimales obtenidas de Médula Ósea, Tejido Adiposo y Gelatina de Wharton en base a los Criterios de la ISCT. Revista Mexicana de Ingeniería Biomédica. 38(1 [S.I.]):280-7. DOI: dx.doi.org/10.17488/RMIB.38.1.23

[11] Erices A, Conget P, Minguell JJ. (2000). Mesenchymal progenitor cells in human umbilical cord blood. Br J Haematol. 109(1):235-42. DOI: bjh1986 [pii]

[12] Roufosse C.A., et al., (2014). Circulating mesenchymal stem cells. Int J Biochem Cell Biol. 36(4):585-97.

DOI: 10.1016/j.biocel.2003.10.007

[13] Meng X, et al. (2007). Endometrial regenerative cells: a novel stem cell population. J Transl Med. 15;5:57.

DOI: 10.1186/1479-5876-5-57

[14] Gronthos S, et al., (2000). Postnatal human dental pulp stem cells (DPSCs) in vitro and in vivo. Proc Natl Acad Sci US. 5;97(25):1362530. DOI: 10.1073/pnas.240309797

[15] Sessarego N., et al. (2008). Multipotent mesenchymal stromal cells from amniotic fluid: solid perspectives for clinical application. Haematologica. 93(3):339-46. DOI: 10.3324/haematol.11869

[16] Yan X.L., et al. (2012). Mesenchymal stem cells from primary breast cancer tissue promote cancer proliferation and enhance mammosphere formation partially via EGF/EGFR/Akt pathway. Breast Cancer Res Treat. 132(1):153-64.

DOI: 10.1007/s10549-011-1577-0
[17] Aktas M., et al. (2010). Good manufacturing practice-grade production of unrestricted somatic stem cell from fresh cord blood. Cytotherapy. 12(3):338-48. DOI: 10.3109/14653241003695034

[18] Manochantr S, et al. (2013). Immunosuppressive properties of mesenchymal stromal cells derived from amnion, placenta, Wharton's jelly and umbilical cord. Intern Med J. 43(4):430-9. DOI: $10.1111 / \mathrm{imj} .12044$

[19] Semont A., et al., (2013). Mesenchymal stem cell therapy stimulates endogenous host progenitor cells to improve colonic epithelial regeneration. PLoS ONE. 8(7):e70170. DOI: 10.1371/journal.pone.0070170

[20] Augustin M, and Vanscheidt W. (2012). Chronic venous leg ulcers: the future of cell-based therapies. Lancet. 15;380(9846):953-5. DOI: $10.1016 / \mathrm{S0140-6736(12)61255-0}$

[21] Zollino I., et al. (2017). Autologous adipose-derived stem cells: Basic science, technique, and rationale for application in ulcer and wound healing. Phlebology. 32(3):160-71. DOI: $10.1177 / 0268355516641546$

[22] Hu J, et al. (2016). Safety and therapeutic effect of mesenchymal stem cell infusion on moderate to severe ulcerative colitis. Exp Ther Med. 12(5):2983-9. DOI: $10.3892 /$ etm.2016.3724 ETM-0-0-3724

[23] Zeng X., et al. (2017). Three-week topical treatment with placentaderived mesenchymal stem cells hydrogel in a patient with diabetic foot ulcer: A case report. Medicine (Baltimore). Dec;96(51):e9212. DOI: 10.1097/MD.0000000000009212

[24] Badiavas E.V., and Falanga V. (2003). Treatment of chronic wounds with bone marrow-derived cells. Arch Dermatol. 139(4):510-6. DOI: 10.1001/archderm.139.4.510 139/4/510

[25] Savoji H, et al., (2018). Skin Tissue Substitutes and Biomaterial Risk Assessment and Testing. Front Bioeng Biotechnol. 6:86. DOI: 10.3389/fbioe.2018.00086

[26] Carriel V., et al., (2018). Ex Vivo and In Vivo Stem Cells-Based Tissue Engineering Strategies for Their Use in Regenerative Medicine. Stem Cells Int. DOI: 10.1155/2018/7143930

[27] Dominici M., et al. (2006). Minimal criteria for defining multipotent mesenchymal stromal cells. The International Society for Cellular Therapy position statement. Cytotherapy. 8(4):315-7. DOI: $10.1080 / 14653240600855905$

[28] Shah SV, Chakravarthy D. (2015). Evaluation of a bovine 100\% native collagen for the treatment of chronic wounds: a case series. J Wound Ostomy Continence Nurs. 42(3):226-234. DOI: 10.1097/WON.0000000000000124

[29] Zhou L., et al. (2018). Efficient differentiation of vascular endothelial cells from dermal-derived mesenchymal stem cells induced by endothelial cell lines conditioned medium. Acta Histochem. 21. DOI:10.1016/j.acthis.2018.08.004

[30] Jiménez-Hernández F., et al., (2014). Tratamiento de úlceras venosas en las extremidades inferiores con autoinjertos en sacabocado más compresión contra compresión elástica. Dermatol Rev Mex 58. www.medigraphic.com/pdfs/derrevmex/rmd-2014-rmd146c.pdf

[31] Ganary D., et. Al., (2013). The emerging use of bone marrowderived mesenchymal stem cells in the treatment of human chronic wounds. Expert Opin. Emerging Drugs 18(4):1-15. 
[32] Benítez-Arvízu G., et al., (2015). Células troncales mesenquimales autólogas e injerto cutáneo autólogo para tratamiento de una úlcera crónica secundaria a diabetes mellitus tipo 2. Cirugía y Cirujanos 83(6):532-536.

[33] Amato B, et al., (2015). The role of adult tissue-derived stem cells in chronic leg ulcers: a systematic review focused on tissue regeneration medicine. Int Wound J. DOI: 10.1111/iwj.12499

[34] Marfia G., et al., (2015). Mesenchymal Stem Cells: Potential For Therapy and Treatment Of Chronic Non-Healing Skin Wounds, Organogenesis, DOI: 10.1080/15476278.2015.1126018

[35] Gadelkarima M., (2018). Adipose-derived stem cells: Effectiveness and advances in delivery in diabetic wound healing. Biomedicine \& Pharmacotherapy 107: 625-633. DOl: doi.org/10.1016/j.biopha.2018.08.013

[36] Francesco De Francesco, et al., (2016). A Regenerative Approach with Dermal Micrografts in the Treatment of Chronic Ulcers. Stem Cell Rev and Rep. DOI: 10.1007/s12015-016-9692-2

[37] Austin Nuschke. (2014). Activity of mesenchymal stem cells in therapies for chronic skin wound healing. Organogenesis 10:1, 29-37. DOI: http://dx.doi.org/10.4161/org.27405

[38] Etulain J. Platelets in wound healing and regenerative medicine. Platelets. 2018 Sep;29(6):556-68. DOI: 10.1080/09537104.2018.1430357

[39] Abou-Elela A, et al., (2009). Use of oxidized cellulose hemostats (Surgicel) to support parenchymal closure and achieve hemostasis following partial nephrectomy. Surg Technol Int. 18:75-9.

[40] Chen S., et al., (2015). Mesenchymal stem cell-laden antiinflammatory hydrogel enhances diabetic wound healing. Scientific Reports 5:18104. DOI: 10.1038/srep18104

[41] Li X., et al., (2014). Comprehensive characterization of four different populations of human mesenchymal stem cells as regards their immune properties, proliferation and differentiation. Int J Mol Med. 34(3):695-704. DOI: 10.3892/ijmm.2014.1821

[42] Caplan AI. (1991). Mesenchymal stem cells. J Orthop Res. 9(5):64150. DOI: $10.1002 /$ jor. 1100090504

[43] Sasaki M., et al. (2008). Mesenchymal stem cells are recruited into wounded skin and contribute to wound repair by transdifferentiation into multiple skin cell type. J Immunol. 15;180(4):2581-7. DOI: 180/4/2581 [pii]

[44] Yeh E.T.H., et al., (2003). Transdifferentiation of human peripheral blood CD34(+)-enriched cell population into cardiomyocytes, endothelial cells, and smooth muscle cells in vivo. Circulation. 28;108(17):2070-3. DOI: 10.1161/01.Cir.0000099501.52718.70

[45] Yianni V, Sharpe PT. (2018). Molecular programming of perivascular stem cell precursors. Stem Cells. DOI: 10.1002/stem.2895

[46] Wang Y., et al., (2018). Effect of Deltex-1 on proliferation and differentiation of bone marrow mesenchymal stem cells into smooth muscle cells. Eur Rev Med Pharmacol Sci. 22(12):3627-34. DOI: 10.26355/eurrev_201806_15239

[47] Li Y., et al. (2017). Differentiation of Human Amniotic Mesenchymal Stem Cells into Human Anterior Cruciate Ligament Fibroblast Cells by In Vitro Coculture. Biomed Res Int. DOI: $10.1155 / 2017 / 7360354$

[48] Soundararajan M, and Kannan S. (2018). Fibroblasts and mesenchymal stem cells: Two sides of the same coin? J Cell Physiol. DOI: 10.1002/jcp.26860. 794
[49] Chowdhury R., (2015). Cancer exosomes trigger mesenchymal stem cell differentiation into pro-angiogenic and pro-invasive myofibroblasts. Oncotarget. 20;6(2):715-31. DOI: 2711 [pii], 10.18632/oncotarget.2711

[50] Linard C., et al. (2018). Autologous Bone Marrow Mesenchymal Stem Cells Improve the Quality and Stability of Vascularized Flap Surgery of Irradiated Skin in Pigs. Stem Cells Transl Med. 7(8):56982. DOI: $10.1002 / \mathrm{sctm} \cdot 17-0267$

[51] Lecarpentier Y., et al. (2018). Human Bone Marrow Contains Mesenchymal Stromal Stem Cells That Differentiate In Vitro into Contractile Myofibroblasts Controlling T Lymphocyte Proliferation. Stem Cells Int. DOI: 10.1155/2018/6134787

[52] Steffens D., et al., (2015). Development of a biomaterial associated with mesenchymal stem cells and keratinocytes for use as a skin substitute. Regen Med. 2015 Nov;10(8):975- 87. DOI: $10.2217 /$ rme.15.58

[53] Seidelmann SB., et al., (2014). Development and pathologies of the arterial wall. Cell Mol Life Sci. Jun;71(11):1977-99. DOI: $10.1007 / \mathrm{s} 00018-013-1478-y$

[54] Raffetto JD. (2016). Pathophysiology of wound healing and alterations in venous leg ulcers-review. Phlebology. Mar;31(1 Suppl):56-62. DOI: 10.1177/0268355516632998

[55] Blaise S., et. Al., (2017). Roustit M, Forli A, Imbert B, Cracowski JL. Non-healing ischaemic digital ulcer in a systemic sclerosis patient: a challenging clinical case. Int Wound J. 14(6):978-81. DOI: 10.1111/iwj.12742

[56] Fiordaliso F, (2016). Prospective study on microangiopathy in type 2 diabetic foot ulcer. Diabetologia. 59(7):1542-1548.

DOI: 10.1007/s00125-016-3961-0

[57] Li X, et. Al., (2018). Exosomes from adipose-derived stem cells overexpressing Nrf2 accelerate cutaneous wound healing by promoting vascularization in a diabetic foot ulcer rat model. Exp Mol Med. 13;50(4):29. DOI: 10.1038/s12276-018-0058-5

[58] Payushina OV, (2018). Effect of Mesenchymal Stromal Cells and Conditioned Media on Healing of Skin Wound. Bull Exp Biol Med. 165(4):572-5. DOI: 10.1007/s10517-018-4215-6

[59] Navone et al. (2014). Decellularized silk fibroin scaffold primed with adipose mesenchymal stromal cells improves wound healing in diabetic mice. Stem Cell Research \& Therapy 5:7. http:// stemcellres.com/content/5/1/7

[60] Mineda Kazohide M., (2015). Therapeutic Potential of Human Adipose-Derived Stem/Stromal Cell Microspheroids Prepared by Three-Dimensional Culture in Non-Cross-Linked Hyaluronic Acid Gel. Stem Cells Traslational Medicine 4:1511-1522. DOI: http:// dx.doi.org/10.5966/sctm.2015-0037

[61] Wu SC, et al., (2017). Safety and efficacy of intramuscular human placenta-derived mesenchymal stromal-like cells (cenplacel [PDA002]) in patients who have a diabetic foot ulcer with peripheral arterial disease. Int Wound J. 14:823-829.

[62] Ribeiro J., et al., (2014). Cell Therapy with Human MSCs Isolated from the Umbilical Cord Wharton Jelly Associated to a PVA Membrane in the Treatment of Chronic Skin Wounds. . Int. J. Med. Sci. 11(10): 979-987. DOI: 10.7150/ijms.9139 